Subject Section

\title{
Drug repurposing through joint learning on knowledge graphs and literature
}

\author{
Mona Alshahrani ${ }^{1}$ and Robert Hoehndorf ${ }^{1, *}$ \\ ${ }^{1}$ Computer, Electrical and Mathematical Sciences and Engineering Division, Computational Bioscience Research Center, King \\ Abdullah University of Science and Technology, Thuwal 23955, Saudi Arabia. \\ *To whom correspondence should be addressed. \\ Associate Editor: $\mathrm{XXXXXXX}$ \\ Received on XXXXX; revised on XXXXX; accepted on XXXXX
}

\section{Abstract}

\section{Motivation:}

Drug repurposing is the problem of finding new uses for known drugs, and may either involve finding a new protein target or a new indication for a known mechanism. Several computational methods for drug repurposing exist, and many of these methods rely on combinations of different sources of information, extract hand-crafted features and use a computational model to predict targets or indications for a drug. One of the distinguishing features between different drug repurposing systems is the selection of features. Recently, a set of novel machine learning methods have become available that can efficiently learn features from datasets, and these methods can be applied, among others, to text and structured data in knowledge graphs.

Results: We developed a novel method that combines information in literature and structured databases, and applies feature learning to generate vector space embeddings. We apply our method to the identification of drug targets and indications for known drugs based on heterogeneous information about drugs, target proteins, and diseases. We demonstrate that our method is able to combine complementary information from both structured databases and from literature, and we show that our method can compete with well-established methods for drug repurposing. Our approach is generic and can be applied to other areas in which multi-modal information is used to build predictive models.

Availability: https://github.com/bio-ontology-research-group/multi-drug-embedding Contact: robert.hoehndorf@kaust.edu.sa

\section{Introduction}

The process of finding a new drug that binds a specific protein or can be used to treat a specific disease is usually time consuming and costly, taking many years and often millions of dollar (Paul et al., 2010). In response, several computational approaches have been developed to identify drug targets and indications for known drugs (Chen et al., 2015; Pryor and Cabreiro, 2015). Many of these approaches utilize the large volumes of data that have become available in the public domain about chemical compounds, drug and protein structures, or target sequences.

Computational drug repurposing and drug discovery methods include chemoinformatics-based methods, network-based methods, and methods based on data- or text-mining. Chemoinformatics-based methods include panel docking and ligand-based approaches (Katsila et al., 2016; Meng et al., 2011) which often rely on knowledge or predictions about the tertiary structure of the target protein. Network-based approaches for drug repurposing utilize different data sources, including genomic and chemical similarities and various other drugs and proteins interactions profiles or descriptors (Yamanishi et al., 2008; Wang et al., 2014a), integrate information related to drug mechanisms, and use machine learning techniques or graph inference methods to predict novel drug targets (Seal et al., 2015; Fu et al., 2016; Chen et al., 2012). Additionally, omics data, in particular gene expression has been used for analyzing or inferring new drugs indications (Subramanian et al., 2017). Some approaches to drug repurposing rely on data- and text-mining and are based on identification of patterns in databases or natural language text to predict novel associations between drugs and targets or drugs and diseases (Swanson, 1990; Andronis et al., 2011; Frijters et al., 2010; Agarwal and Searls, 2008). 
The different computational approaches to drug repurposing diffe both in the algorithms they employ as well as in the data sources they utilize. Finding innovative ways to use novel kinds of data or combine different types of multi-modal information in a single model has the potential to significantly improve predictive performance. For example, the PREDICT method (Gottlieb et al., 2011) combined several different types of data, including similarity between proteins based on their sequence and based on their functions, as well as similarity between drugs based on their side effects or their targets. Other approaches combine disease genes associations, drugs targets, signaling pathways, and gene expression profiles to discover new therapeutic roles for known drugs (Peyvandipour et al., 2018).

Many types of data that are relevant for drug repurposing can be integrated through Semantic Web technologies (Berners-Lee et al., 2001), notably using the Resource Description Framework (RDF) (Manola et al. 2004) and ontologies formalized in the Web Ontology Language (OWL) (Grau et al., 2008). Initiatives that aim to integrate knowledge relevant for drug discovery and drug repurposing include the Open PHACTS (Williams et al., 2012) and Bio2RDF (Belleau et al., 2008) projects, and other relevan RDF datasets are made available by the European Bioinformatics Institute (EBI)(Jupp et al., 2014).

Recently, several unsupervised machine learning methods have become available that can learn feature representations of entities represented in different types of data (LeCun et al., 2015). For unstructured text, vector space models such as Word2Vec (Mikolov et al., 2013) or GLOVE (Pennington et al., 2014) can learn representations of words that preserve some the words' semantics under certain vector operations and can therefore be used to build predictive models. Similar methods have been developed for information represented as graphs (Perozzi et al., 2014), knowledge graphs (Nickel et al., 2016b), or formal knowledge bases (Gutiérrez-Basulto and Schockaert, 2018), and these methods have led to the development of machine learning models that can significantly outperform classic predictive methods (Alshahrani et al., 2017).

Combining different types of data in a single predictive model has the potential to increase the performance of computational drug repurposing methods. Such a combination can either be done by applying differen method individually on different types of data and utilize their prediction results in a new model, or by combining the different approaches within a single model.

Here, we present an approach to systematically integrate multi-modal information from knowledge graphs and literature in predictive machine learning models. Specifically, our approach utilizes structured, semantic information that can be represented in knowledge graphs and combine this information with features extracted from unstructured text. We apply the resulting combined features for drug repurposing. Using supervised machine learning, we demonstrate that our approach and the resulting model outperforms the use of individual features and leads to significant improvements when applied to computational drug repurposing.

\section{Methods}

\subsection{Data sources}

We use a knowledge graph containing information about genes/proteins, drugs, diseases, and functions generated to demonstrate the utility of knowledge graph embedding methods in life sciences (Alshahran et al., 2017). The knowledge graph is illustrated in Supplementary Figure 1. This graph consists of three ontologies, the Gene Ontology (GO) (Ashburner et al., 2000), Disease Ontology (DO) (Schriml et al., 2011), and the Human Phenotype Ontology (HPO) (Köhler et al. 2014). It also includes three types of biological entity: diseases, genes or proteins (we do not distinguish between them in our graph), and chemicals or drugs, as well as their interactions or associations with ontology classes. The graph further includes relations between entities such as the interactions between genes/proteins obtained from STRING (Szklarczyk et al., 2011) (file protein.actions.v10.txt.gz), chemical-protein interactions from STITCH (Kuhn et al., 2012) (file 9606.actions.v4.0.tsv), and drugs and their indications from SIDER (Kuhn et al., 2015) (file meddra_all_indications.tsv). We built the graph using RDF and downloaded all evaluation data on 11 March 2018.

For text processing, we use the pre-annotated Medline corpus provided by the PubTator project (Wei et al., 2013), downloaded on 18 Dec 2017. This corpus contains 27,599,238 abstracts together with annotations to chemicals, genes/proteins, and diseases. We use the annotations provided by PubTator for chemicals, genes/proteins, and diseases. PubTator has annotations to 17,505,118 chemicals mentions covering 129,085 distinct drugs using either CHEBI or MESH identifiers. We could map 9,545 of these to STITCH identifier using the file 9606 . protein.aliases.v10.txt provided by STITCH. PubTator further contains 81,655,248 disease mentions covering 8,143 distinct diseases in MESH. We use the DO and map these to 2,581 distinct DO classes. Furthermore, PubTator contains 17,260,141 gene/protein mentions covering 137,353 distinct genes in different species, 35,466 of which refers to human genes.

\subsection{Generation of corpus and text normalization}

We use an edge-labeled iterated random walk of fixed length and without restart to generate a corpus from the knowledge graph (Alshahrani et al., 2017). For each vertex in the graph, we generate a sentence based on a short random walk. Each walk is a sequence of tokens, i.e., nodes and edges. We have two parameters for corpus generation: walk length and number of walks. Walk length is the size of each walk sequence and the number of walks is the total number of walks generated for each vertex. For all experiments, we use a walk length of 20 and perform 50 random walks for each node.

\subsection{Learning Embeddings}

We use Word2Vec (Mikolov et al., 2013) to generate embeddings for entities in our knowledge graph and for words found in text. Word2vec is a vector space model mapping words to vectors based on the co-occurrence of a word with other words within a context window across a corpus of text. In our graph, this semantics is captured by the random walks representing the co-occurrence of different entities and relations. We use the skip-gram model (Mikolov et al., 2013) in Word2Vec on the corpus generated by random walks on the knowledge graph. As parameters for both corpora, we use negative sampling using 5 words drawn from the noise distribution, a window size of 10 , and an embedding size of 128 .

\subsection{Training of supervised prediction models}

We evaluated the performance of each embedding method by using the embedding vectors to predict drug-target or drug-disease associations in a supervised manner. As prediction models, we use artificial neural networks, random forests, and logistic regression. For training the neural networks model, we used an architecture with a single hidden layer consisting of twice the size of the input features vector. We use a Rectified Linear Unit (ReLU) (Nair and Hinton, 2010) as an activation function for the hidden layer and a sigmoid function as the activation function for the output layer; we use cross entropy as loss function in training, and Rmsprop (Hinton et al., 2012) to optimize the neural networks parameters during training. For training the neural networks, we used the Keras library 


$$
\text { “main" - 2018/8/6 - 13:28 - page 3 - \#3 }
$$

in Python (Chollet et al., 2015). For training the random forest classifier, we specified the number of trees to be 50 , with the minimum number of training samples in leaf nodes to be one, and Gini impurity index to measure the quality of the split. For the logistic regression classifier, we used the default settings of scikit-learn in Python (Pedregosa et al., 2011).

\subsection{Multi-modal drug repurposing}

We compare the performance of our method using a validation strategy in which we randomly remove a set of associations and measure how well our method can reproduce these. For each interaction type (drug-target or drug-disease associations), we remove all interactions from our knowledge graph before generating embeddings. We then use $80 \%$ of the associations to train a classifier and we test and evaluate our method on the remaining $20 \%$. We identify the $20 \%$ randomly among all interactions. Furthermore, we randomly select an equal number of weak negative interactions, i.e., pairs of a drug and gene/protein (when predicting targets) or disease (when predicting interactions) that are not known to be associated, and we use these as negatives during training of our classifiers.

For the rank-based evaluation (ROCAUC or recall at certain ranks), we create an embedding matrix for each drug in which we fix the first part of the matrix to represent a particular drug embedding and the second part represents the gene/protein or disease embedding. We then apply the learned model on the matrix and rank the genes/proteins or diseases based on the confidence scores provided by the classifiers.

The true positive rate (TPR) and false positive rate (FPR) at each rank are used to identify the proportion of correctly and falsely predicted interactions. We quantify the performance of the predictions through the area under the receiver operating characteristic (ROC) curve (Fawcett, 2006). A ROC curve is a plot of the TPR as a function of the FPR. The TPR at a particular rank is defined as a rate of correctly predicted drugtarget interactions or drug-disease associations, and the FPR is the rate of predicted interactions that are not drug-target or drug-disease interactions. As we do not have true negative drug-target interactions, we use "weak" negatives and treat all unassociated pairs of drug and gene/protein or disease as negatives. The recall at ranks ten and 100 is calculated as the ratio of predicted true positives in the top ten or 100 over the total number of positives.

\section{Results}

\subsection{Integrating literature and structured knowledge}

Information about drugs and their targets is present in several locations and formats, including in structured databases and in scientific literature. We base our approach on an integrated dataset consisting of structured data from multiple databases and literature. We use the Resource Description Framework (RDF) (Beckett, 2004) to express and integrate structured information we consider useful for predicting drug-target and drugindications associations. In RDF, knowledge is expressed in a graph-based format in which entities are represented by an Internationalized Resource Identifier (IRI) and relations between entities as a property that connects two nodes.

We integrate several datasets related to drug actions and diseases in a knowledge graph using RDF as representation language. Specifically, we combine information about drugs and their targets (Kuhn et al., 2012) and indications (Kuhn et al., 2015), gene-disease associations (Piñero et al., 2017), and disease phenotypes (Hoehndorf et al., 2015), as well as gene functions and interactions between gene products (Szklarczyk et al., 2011). For example, we link the disease Primary pulmonary hypertension (DOID:14557) to the phenotype Arrhythmia (HP:0011675) (using a has phenotype relation), we link the gene CAV1 to disease Primary pulmonary hypertension (DOID : 14557) (using a has disease association relation), and we link the drug Tadalafil (CID00110635) to phenotype Abdominal pain (HP : 0002027) (using a has sideeffect relation):

@prefix doid: <http://purl.obolibrary.org/obo/DOID_> eprefix hp: <http://purl.obolibrary.org/obo/HP_> @prefix b2v: <http://bio2vec.net/relation/>. @prefix entrez: <http://www.ncbi.nlm.nih.gov/gene/> . @prefix stitch: <http://bio2vec.net/CID> .

doid:14557 b2v:has_disease_phenotype hp:0011675. entrez:857 b2v:has_disease_association doid:14557. stitch:00110635 b2v:has_sideeffect hp:0002027. stitch:00110635 b2v:has_indication doid:65.

We further add biological background knowledge expressed in ontologies, specifically the Human Phenotype Ontology (HPO) (Köhler et al., 2014), Gene Ontology (GO) (Ashburner et al., 2000) and Disease Ontology (DO) (Schriml et al., 2011), directly to this RDF graph so that the superclasses of phenotypes can be accessed and used during machine learning. Supplementary Figure 1 shows the graph we build and the relations between the different biological entities it includes.

To learn representations of features contained in this knowledge graph, we apply a random walk algorithm over RDF and OWL (Alshahrani et al., 2017) and generate a corpus consisting of iterated random walks through this graph, starting from each node. We consider each random walk as a "sentence" that expresses a chain of statements following a random path through the knowledge graph.

As next step, we integrate the information in our knowledge graph with information contained in biomedical literature. For this purpose, we normalize biomedical literature abstracts to our knowledge graph using named entity recognition and entity normalization approaches (RebholzSchuhmann et al., 2012) that were developed for the entities in our graph. Specifically, we normalize drug, gene, and disease mentions to our graph using the literature annotations of PubMed abstracts provided by the PubTator (Wei et al., 2013) database as well as mappings provided between different vocabularies of drugs and diseases (see Methods). PubTator aggregates different entity normalization approaches such as GNorm (Wei et al., 2015) or DNorm (Leaman et al., 2013), which can also be used directly with new text.

As a next step, we process the annotated corpus of PubMed abstracts by replacing each mention of an entity (i.e., gene, chemical compounds, or disease) that is also included in our knowledge graph with the IRI used to represent the entity in the knowledge graph. Specifically, we ensure that the mentions in text that can be normalized to our knowledge graph are "token-identical" to the entities we represent in our knowledge graph (and the corpus resulting from our random walks through the graph). This replacement ensures that our text corpus and knowledge graph overlap on the level of tokens, and we can use this overlap in our machine learning models. Figure 1 illustrates the normalization step between the text corpus and knowledge graph.

As an end result of these processing steps we have generated two corpora: one consisting of random walks starting from nodes in our knowledge graph, and another consisting of literature abstracts in which the mentions of entities that also appear in our graph have been replaced by the entities' IRI in the graph. These two corpora form the foundation of our feature learning step.

\subsection{Learning and combining features}

Biological literature and the information in our knowledge graph will contain different information, and our aim is to establish a way to combine information in both data sources within a single predictive model. To achieve this goal, we first apply an unsupervised machine learning 


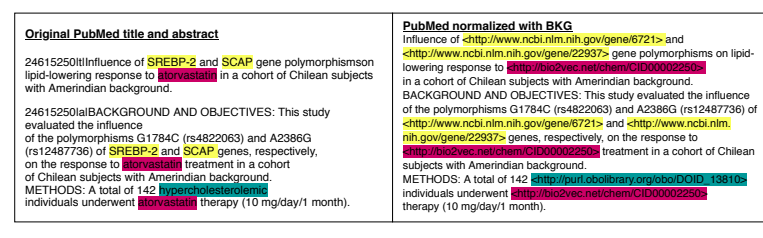

Fig. 1. Illustration of how we normalize literature abstracts to our knowledge graph to ensure that both overlap on the level of tokens.

approach to generate embeddings for entities in our knowledge graph as well as for the words in our text corpus. An embedding encodes within a vector space the context in which an entity appears; since we represent information about entities in the knowledge graph through sentences generated from random walks, we apply the Word2Vec skip-gram model (Mikolov et al., 2013) to generate embeddings for all terms that occur within the two corpora we generated.

We use two different approaches to combine the embeddings from the knowledge graph and text corpus: first, we generate embeddings independently on both corpora, and concatenate the resulting embedding vectors; and second, we concatenate the two corpora and generate embeddings from the combined corpus. As a result, we obtain embedding for drugs, genes, diseases, and for all other entities from the knowledge graph; we further obtain embeddings for all words that are used in our literature corpus, in particular for the entities which are mentioned in literature and which we normalized to our knowledge graph. However, not all entities in our knowledge graph also have a representation in literature, and not all entities (drugs, diseases, and genes) mentioned in literature are included in our knowledge graph. Supplementary Figure 2 shows the overlap between the two datasets. The embedding vectors generated for the entities from the two different corpora (either individually or jointly) form our entities' feature representations, and the features either represent information from structured databases, from literature, or both Supplementary Figure 3 and 4 show a visualization of the embeddings (from the knowledge graph, literature, and combined) using t-SNE (van der Maaten and Hinton, 2008), and coloring disease embeddings based on their top-level DO class, and drug embeddings based on their top-level class in the Anatomical Therapeutic Chemical (ATC) Classification System.

\subsection{Evaluation on prediction of drug targets and indications}

Our method combines heterogeneous information from text and a knowledge graph in vector space embeddings, and we evaluate the performance of our method by predicting drug targets and drug indications. For this purpose, we use four different evaluation methods: first, we use the embeddings generated from the knowledge graph alone; second, we use the embeddings generated from our literature corpus alone; third, we concatenate the embeddings from the knowledge graph and text corpus; and finally, we combine the text corpus and knowledge graph corpus and learn joint embeddings.

We use as evaluation sets the drug targets from the STITCH database (Kuhn et al., 2012) and drug indications from the SIDER database (Kuhn et al., 2015). Furthermore, to clearly distinguish and evaluate the contributions of the different data sources, we initially limit our evaluation set to the drugs, targets, and diseases that have a representation both in our knowledge graph and in our literature corpus. For predicting drug targets, we use as evaluation set 820 drugs and rank 17,380 genes that are both in our knowledge graph and found in the literature corpus. For predicting drug indications, we use 754 drugs with one or more known indications and rank 2,552 diseases (overlapping between literature and our knowledge graph) for each of the drugs to determine for which disease it may be indicated (see Supplementary Tables 1 and 2 for details).

To predict associations between drugs and their targets or drugs and the diseases they may treat, we use supervised machine learning and train a model based on $80 \%$ randomly chosen drug-target or drug-disease associations and test whether the model is able to predict the remaining $20 \%$. We use three different machine learning approaches for the model construction: logistic regression, a random forest classifier, and artificial neural networks. Before training the model, we remove all has-target (when predicting drug targets) or has-indication (when predicting drug indications) edges in the graph before generating the corpus for predicting drug targets and indications, respectively. Each model has as input two embedding vectors that represent a drug and another embedding vector representing either a gene/protein (when predicting targets) or disease (when predicting indications). The models are trained as binary classifiers and output whether the drug targets the gene/protein or treats the disease. Figure 2 provides an overview over our overall workflow.

We evaluate the performance of each model on the $20 \%$ of associations we withhold from training. All three of our classification models can provide confidence values for a prediction, and we rank predicted associations based on their confidence value. We then calculate the area under the receiver operating characteristic (ROC) curve (ROCAUC) (Fawcett, 2006) as well as the number of correct associations we retrieve within the first ten and first 100 ranks. Table 1 summarizes our results for predicting associations between drugs and targets, and Table 2 summarizes the results for predicting indications.

We find that both our artificial neural network and the random forest classifier are able to accurately predict both drug targets and drug indications, while the logistic regression classifier results in relatively worse performance. An obvious explanation is that logistic regression mainly assigns weights to individual features and does not have the ability to compare or match elements of the two input embedding vectors, while both the random forest classifier and an artificial neural network are able to provide a classification based on comparing elements of the input embedding vectors. Furthermore, we find that, in general, using embeddings generated from literature results in higher predictive performance across all classifiers compared to embeddings generated from the knowledge graph alone. Combining the embeddings, either through concatenation of the embeddings or through concatenation of the two corpora sometimes but not always improves or changes the predictive performance.

While our results indicate that both literature-derived and knowledge graph embeddings can be used to predict interactions, the main contribution of our multi-modal approach is the increased coverage through combining database content and literature (see Supplementary Figure 2). To demonstrate this application of our method, we extend our evaluation set to contain all the drugs, genes, and diseases that are found in either our knowledge graph, the literature abstract, or the union of the entities in the knowledge graph and literature trained on the combined corpus. Figure 3 shows the ROC curves and the ROCAUC for predicting drug targets and drug indications using our neural network classifier, based on a combination of the literature corpus and the random walk corpus.

Our knowledge graph contains a very large number of chemicals, many of which are not drug-like, and while the performance in predicting drug targets is somewhat higher when using the knowledge graph embeddings, the overall performance is still dominated by the literature-derived embedding vectors. However, when predicting indications for known drugs, both our graph and literature overlap 


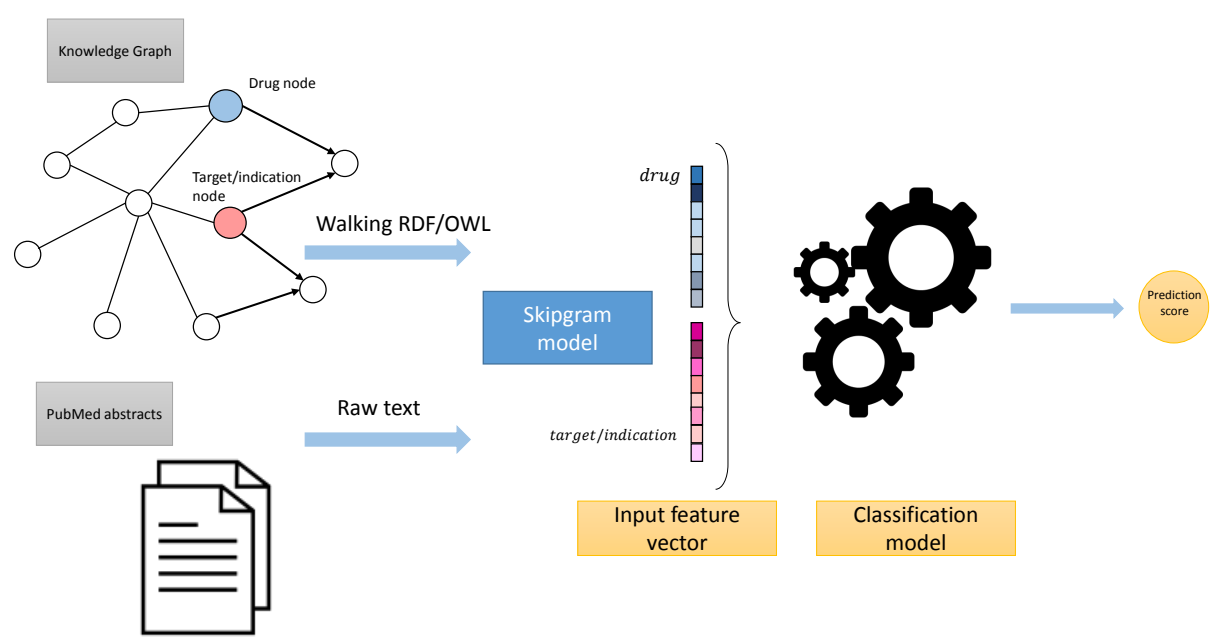

Fig. 2. High-level overview over our workflow.

more substantially while nevertheless containing complementary information. We observe a significant improvement in predicting drug indications when combining the information from literature and the knowledge graph. We make all predictions of drug-target associations as well as the predictions for drug indications freely available athttps://github.com/bio-ontology-research-group/ multi-drug-embedding.

\begin{tabular}{|l|l|l|l|l|}
\hline Model & $\begin{array}{l}\text { Embedding } \\
\text { Method }\end{array}$ & ROCAUC & Recall @ 100 & Recall @ 10 \\
\hline \multirow{4}{*}{ ANN } & Knowledge graph & 0.887 & 0.35 & 0.09 \\
\cline { 2 - 5 } & PubMed abstracts & 0.880 & 0.45 & 0.11 \\
\cline { 2 - 5 } & $\begin{array}{l}\text { Concatenated } \\
\text { embeddings }\end{array}$ & 0.905 & 0.50 & 0.14 \\
\cline { 2 - 5 } & $\begin{array}{l}\text { Concatenated } \\
\text { corpus }\end{array}$ & 0.896 & 0.45 & 0.13 \\
\hline \multirow{5}{*}{ RF } & Knowledge graph & 0.860 & 0.36 & 0.08 \\
\cline { 2 - 5 } & PubMed abstracts & 0.852 & 0.49 & 0.18 \\
\cline { 2 - 5 } & $\begin{array}{l}\text { Concatenated } \\
\text { embeddings }\end{array}$ & 0.867 & 0.45 & 0.13 \\
\cline { 2 - 5 } & $\begin{array}{l}\text { Concatenated } \\
\text { corpus }\end{array}$ & 0.879 & 0.51 & 0.18 \\
\hline \multirow{5}{*}{ LR } & Knowledge graph & 0.837 & 0.10 & 0.01 \\
\cline { 2 - 5 } & PubMed abstracts & 0.822 & 0.20 & 0.04 \\
\cline { 2 - 5 } & $\begin{array}{l}\text { Concatenated } \\
\text { embeddings }\end{array}$ & 0.854 & 0.21 & 0.06 \\
\cline { 2 - 5 } & $\begin{array}{l}\text { Concatenated } \\
\text { corpus }\end{array}$ & 0.837 & 0.22 & 0.05 \\
\hline \multirow{2}{*}{ Table 1. Performance results for predicting drug-target associations, based }
\end{tabular}

\begin{tabular}{|l|l|l|l|l|}
\hline Model & $\begin{array}{l}\text { Embedding } \\
\text { Method }\end{array}$ & ROCAUC & Recall @ 100 & Recall @ 10 \\
\hline \multirow{5}{*}{ ANN } & Knowledge graph & 0.884 & 0.46 & 0.10 \\
\cline { 2 - 5 } & PubMed abstracts & 0.928 & 0.63 & 0.26 \\
\cline { 2 - 5 } & $\begin{array}{l}\text { Concatenated } \\
\text { embeddings }\end{array}$ & 0.917 & 0.58 & 0.22 \\
\cline { 2 - 5 } & $\begin{array}{l}\text { Concatenated } \\
\text { corpus }\end{array}$ & 0.929 & 0.63 & 0.22 \\
\hline \multirow{5}{*}{ RF } & Knowledge graph & 0.895 & 0.44 & 0.13 \\
\cline { 2 - 5 } & PubMed abstracts & 0.912 & 0.61 & 0.24 \\
\cline { 2 - 5 } & $\begin{array}{l}\text { Concatenated } \\
\text { embeddings }\end{array}$ & 0.908 & 0.54 & 0.17 \\
\cline { 2 - 5 } & $\begin{array}{l}\text { Concatenated } \\
\text { corpus }\end{array}$ & 0.918 & 0.60 & 0.22 \\
\hline \multirow{5}{*}{ LR } & Knowledge graph & 0.842 & 0.30 & 0.06 \\
\cline { 2 - 5 } & PubMed abstracts & 0.846 & 0.40 & $: 0.11$ \\
\cline { 2 - 5 } & $\begin{array}{l}\text { Concatenated } \\
\text { embeddings }\end{array}$ & 0.862 & 0.39 & 0.09 \\
\cline { 2 - 5 } & $\begin{array}{l}\text { Concatenated } \\
\text { corpus }\end{array}$ & 0.858 & 0.39 & 0.12 \\
\hline
\end{tabular}

Table 2. Performance results for prediction of drug indications, based on our
four embeddings approaches and using three classification models (Artificial Neural Networks (ANN), Random Forest (RF) and Logistic regression (LR)).

\section{Discussion}

There are many scenarios in biological and biomedical research in which predictive models need to be built that can utilize information that is represented in different formats. Our key contribution is a method to integrate data represented in structured databases, in particular knowledge graphs represented in RDF and OWL, and integrate this information with information in literature. While we primarily focus on the prediction of drug-target interactions and drug indications based on information on our four embeddings approaches and using three classification model (Artificial Neural Networks (ANN), Random Forest (RF) and Logistic regression (LR)).

in text and databases, our approach is generic and can serve as a paradigm for learning from multi-modal, heterogeneous data in biology and biomedicine.

Our method uses feature learning to project different types of data into a vector space, and combine data of different modes either within a single vector space (when mapping data of different modes to the same space, or 

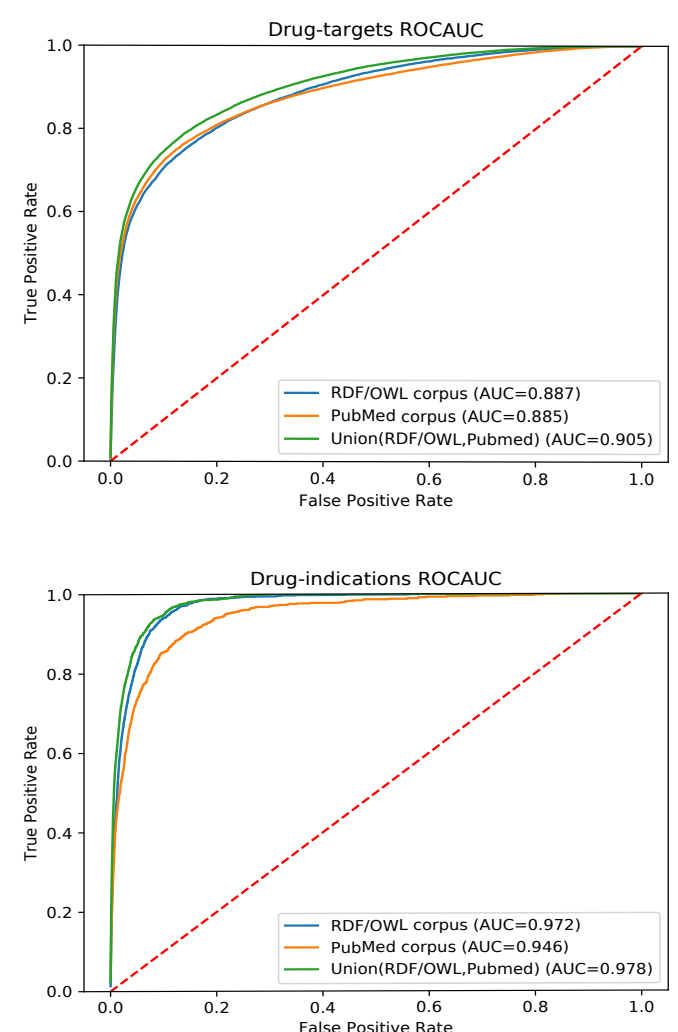

Fig. 3. ROC curve of our neural network for predicting drug targets in the union of associations present in the knowledge graph and PubMed abstracts (top); ROC curve of our neural network for predicting drug indications found in the union of knowledge graph and PubMed abstracts.

to vector spaces of identical dimensions) or we combine the vector spaces themselves. We rely on the recent success of deep learning methods (Ravì et al., 2017; Angermueller et al., 2016) which improved our ability to learn relevant features from a data set and project them into a vector space. In particular, our approach relies on natural language models, in particular Word2Vec (Mikolov et al., 2013), and recent approaches to project information in knowledge graphs into vector spaces (Nickel et al., 2016b; Alshahrani et al., 2017; Smaili et al., 2018). These approaches are now increasingly applied in biological and biomedical research (Alshahran and Hoehndorf, 2018) yet often restricted to single types of representation (such as images, genomic sequences, text, or knowledge graphs).

Our approach naturally builds on the significant efforts that have been invested in the development of named entity recognition and normalization methods for many different biological entities (Rebholz-Schuhmann et al. 2012) as well as the effort to formally represent and integrate biological data using Semantic Web technologies (Jupp et al., 2014; Callahan et al. 2013). Several biological data providers now provide their data natively using RDF (Jupp et al., 2014; UniProt Consortium, 2018). Furthermore, many methods and tools have been developed to normalize mentions of biological entities in text to biological databases, for example for mentions of genes and proteins, (Leaman and Gonzalez, 2008; Wei et al., 2015), chemicals (Leaman et al., 2015) as well as diseases (Leaman et al., 2013), and repositories have been developed to aggregate and integrate the annotations to literature abstracts or fulltext articles (Wei et al., 2013, Kim and Wang, 2012). While these methods, tools, and repositories are not commonly designed to normalize mentions of biological entities to a knowledge graph, we demonstrate here how a normalization of text to a knowledge graph can be achieved, and subsequently use the combined information in our multi-modal machine learning approach. Consequently, our method has the potential to increase the value of freely available Linked Data resources and connect them directly to the methods and tools developed for natural language processing and text mining in biology and biomedicine.

In the future, it would be beneficial to develop better entity normalization methods that can directly normalize entity mentions in text to a knowledge graph. We also intend to evaluate the success of our approach on full-text articles so that more information, in particular regarding methods and experimental protocols, can be utilized by our approach. Methodologically, we also intend to apply other knowledge graph embedding methods, in particular translational embeddings (Bordes et al., 2013; Nickel et al., 2016a; Dai and Yeung, 2006), that have previously been combined successfully with textual information (Wang et al., 2014b), and evaluate their performance for prediction of biological relations.

\section{Funding}

This work was supported by funding from King Abdullah University of Science and Technology (KAUST) Office of Sponsored Research (OSR) under Award No. URF/1/3454-01-01, FCC/1/1976-08-01, and FCS/1/3657-02-01.

\section{References}

Agarwal, P. and Searls, D. B. (2008). Literature mining in support of drug discovery. Briefings in bioinformatics, 9(6), 479-492.

Alshahrani, M. and Hoehndorf, R. (2018). Semantic disease gene embeddings (smudge): phenotype-based disease gene prioritization without phenotypes. Bioinformatics. in press.

Alshahrani, M. et al. (2017). Neuro-symbolic representation learning on biological knowledge graphs. Bioinformatics, 33(17), 2723-2730.

Andronis, C. et al. (2011). Literature mining, ontologies and information visualization for drug repurposing. Briefings in bioinformatics, 12(4), 357-368.

Angermueller, C. et al. (2016). Deep learning for computational biology. Molecular Systems Biology, 12(7).

Ashburner, M. et al. (2000). Gene ontology: tool for the unification of biology. Nature Genetics, 25(1), 25-29.

Beckett, D. (2004). RDF/XML syntax specification (revised). W3C recommendation, World Wide Web Consortium (W3C).

Belleau, F. et al. (2008). Bio2rdf: towards a mashup to build bioinformatics knowledge systems. Journal of biomedical informatics, 41(5), 706-716. Berners-Lee, T. et al. (2001). The semantic web. Scientific american, 284(5), 34-43.

Bordes, A. et al. (2013). Translating embeddings for modeling multirelational data. In C. J. C. Burges, L. Bottou, M. Welling, Z. Ghahramani, and K. Q. Weinberger, editors, Advances in Neural Information Processing Systems 26, pages 2787-2795. Curran Associates, Inc.

Callahan, A. et al. (2013). Bio2RDF Release 2: Improved Coverage, Interoperability and Provenance of Life Science Linked Data, pages 200-212. Springer Berlin Heidelberg, Berlin, Heidelberg.

Chen, L. et al. (2012). Predicting anatomical therapeutic chemical (atc) classification of drugs by integrating chemical-chemical interactions and similarities. PloS one, 7(4), e35254.

Chen, X. et al. (2015). Drug-target interaction prediction: databases, web servers and computational models. Briefings in bioinformatics, 17(4), 696-712.

Chollet, F. et al. (2015). Keras. https: //keras.io. 


$$
\text { “main" — 2018/8/6 - 13:28 — page } 7 \text { — } 7
$$

Dai, G. and Yeung, D.-Y. (2006). Tensor embedding methods. In Proceedings of the 21st National Conference on Artificial Intelligence Volume 1, AAAI'06, pages 330-335. AAAI Press.

Fawcett, T. (2006). An introduction to ROC analysis. Pattern Recogn Lett, 27(8), $861-874$

Frijters, R. et al. (2010). Literature mining for the discovery of hidden connections between drugs, genes and diseases. PLoS computational biology, 6(9), e1000943.

Fu, G. et al. (2016). Predicting drug target interactions using meta-pathbased semantic network analysis. BMC bioinformatics, 17(1), 160

Gottlieb, A. et al. (2011). Predict: a method for inferring novel drug indications with application to personalized medicine. Molecular systems biology, 7(1), 496.

Grau, B. et al. (2008). OWL 2: The next step for OWL. Web Semantics: Science, Services and Agents on the World Wide Web, 6(4), 309-322.

Gutiérrez-Basulto, V. and Schockaert, S. (2018). From knowledge graph embedding to ontology embedding: Region based representations of relational structures. arXiv preprint arXiv:1805.10461.

Hinton, G. et al. (2012). Lecture 6a overview of mini-batch gradient descent.

Hoehndorf, R. et al. (2015). Analysis of the human diseasome using phenotype similarity between common, genetic, and infectious diseases. Scientific Reports, 5, 10888.

Jupp, S. et al. (2014). The EBI RDF platform: linked open data for the life sciences. Bioinformatics, 30(9), 1338-1339.

Katsila, T. et al. (2016). Computational approaches in target identification and drug discovery. Computational and structural biotechnology journal, 14, 177-184.

Kim, J.-D. and Wang, Y. (2012). Pubannotation: A persistent and sharable corpus and annotation repository. In Proceedings of the 2012 Workshop on Biomedical Natural Language Processing, BioNLP '12, pages 202205, Stroudsburg, PA, USA. Association for Computational Linguistics. Köhler, S. et al. (2014). The human phenotype ontology project: linking molecular biology and disease through phenotype data. Nucleic Acids Res, 42(D1), D966-D974.

Kuhn, M. et al. (2012). STITCH 3: zooming in on protein-chemical interactions. Nucleic Acids Research, 40(D1), D876-D880.

Kuhn, M. et al. (2015). The SIDER database of drugs and side effects. Nucleic acids research, 44(D1), D1075-D1079.

Leaman, R. and Gonzalez, G. (2008). Banner: an executable survey of advances in biomedical named entity recognition. Pacific Symposium on Biocomputing. Pacific Symposium on Biocomputing, pages 652-663.

Leaman, R. et al. (2013). Dnorm: disease name normalization with pairwise learning to rank. Bioinformatics, 29(22), 2909-2917.

Leaman, R. et al. (2015). tmchem: a high performance approach for chemical named entity recognition and normalization. Journal of cheminformatics, 7(1), S3.

LeCun, Y. et al. (2015). Deep learning. nature, 521(7553), 436.

Manola, F. et al. (2004). Rdf primer. W3C recommendation, 10(1-107), 6.

Meng, X.-Y. et al. (2011). Molecular docking: a powerful approach for structure-based drug discovery. Current computer-aided drug design, 7(2), 146-157.

Mikolov, T. et al. (2013). Distributed representations of words and phrases and their compositionality. In C. J. C. Burges, L. Bottou, M. Welling, Z. Ghahramani, and K. Q. Weinberger, editors, Advances in Neural Information Processing Systems 26, pages 3111-3119. Curran Associates, Inc

Nair, V. and Hinton, G. E. (2010). Rectified linear units improve restricted boltzmann machines. In Proceedings of the 27th international conference on machine learning (ICML-10), pages 807-814.
Nickel, M. et al. (2016a). Holographic embeddings of knowledge graphs. In Proceedings of the Thirtieth AAAI Conference on Artificial Intelligence, AAAI'16, pages 1955-1961. AAAI Press.

Nickel, M. et al. (2016b). A review of relational machine learning for knowledge graphs. Proceedings of the IEEE, 104(1), 11-33.

Paul, S. M. et al. (2010). How to improve r\&d productivity: the pharmaceutical industry's grand challenge. Nature reviews Drug discovery, 9(3), 203.

Pedregosa, F. et al. (2011). Scikit-learn: Machine learning in Python Journal of Machine Learning Research, 12, 2825-2830.

Pennington, J. et al. (2014). Glove: Global vectors for word representation. In Proceedings of the 2014 conference on empirical methods in natural language processing (EMNLP), pages 1532-1543.

Perozzi, B. et al. (2014). Deepwalk: Online learning of social representations. In Proceedings of the 20th ACM SIGKDD international conference on Knowledge discovery and data mining, pages 701-710. ACM.

Peyvandipour, A. et al. (2018). A novel computational approach for drug repurposing using systems biology. Bioinformatics.

Piñero, J. et al. (2017). Disgenet: a comprehensive platform integrating information on human disease-associated genes and variants. Nucleic Acids Research, 45(D1), D833.

Pryor, R. and Cabreiro, F. (2015). Repurposing metformin: an old drug with new tricks in its binding pockets. Biochemical Journal, 471(3), 307-322.

Ravì, D. et al. (2017). Deep learning for health informatics. IEEE Journal of Biomedical and Health Informatics, 21(1), 4-21.

Rebholz-Schuhmann, D. et al. (2012). Text-mining solutions for biomedical research: enabling integrative biology. Nature Reviews Genetics, 13(12), 829-839.

Schriml, L. M. et al. (2011). Disease ontology: a backbone for disease semantic integration. Nucleic acids research, 40(D1), D940-D946.

Seal, A. et al. (2015). Optimizing drug-target interaction prediction based on random walk on heterogeneous networks. Journal of cheminformatics, 7(1), 40

Smaili, F. Z. et al. (2018). Onto2vec: joint vector-based representation of biological entities and their ontology-based annotations. Bioinformatics. in press.

Subramanian, A. et al. (2017). A next generation connectivity map: L1000 platform and the first 1,000,000 profiles. Cell, 171(6), $1437-1452$.

Swanson, D. R. (1990). Medical literature as a potential source of new knowledge. Bulletin of the Medical Library Association, 78(1), 29.

Szklarczyk, D. et al. (2011). The STRING database in 2011: functional interaction networks of proteins, globally integrated and scored. Nucleic Acids Research, 39(suppl 1), D561-D568.

UniProt Consortium, T. (2018). Uniprot: the universal protein knowledgebase. Nucleic Acids Research, 46(5), 2699.

van der Maaten, L. and Hinton, G. (2008). Visualizing data using t-SNE. Journal of Machine Learning Research, 9, 2579-2605.

Wang, B. et al. (2014a). Similarity network fusion for aggregating data types on a genomic scale. Nature methods, 11(3), 333.

Wang, Z. et al. (2014b). Knowledge graph and text jointly embedding. In The 2014 Conference on Empirical Methods on Natural Language Processing. ACL - Association for Computational Linguistics.

Wei, C.-H. et al. (2013). Pubtator: a web-based text mining tool for assisting biocuration. Nucleic Acids Research, 41(W1), W518.

Wei, C.-H. et al. (2015). Gnormplus: an integrative approach for tagging genes, gene families, and protein domains. BioMed research international, 2015.

Williams, A. J. et al. (2012). Open phacts: semantic interoperability for drug discovery. Drug discovery today, 17(21-22), 1188-1198. 
